\title{
Meal fat composition has differential effects on biomarkers of postprandial endothelial function in postmenopausal women
}

\author{
Kumari M. Rathnayake, Michelle Weech, Kim G. Jackson and Julie A. Lovegrove \\ Hugh Sinclair Unit of Human Nutrition and Institute for Cardiovascular and Metabolic Research, Department of Food \\ \& Nutritional Sciences, University of Reading, Reading, UK, RG6 6AP
}

Decline in oestrogen at menopause is associated with adverse effects on lipid metabolism, vascular function and blood pressure ${ }^{(1)}$, markedly increasing cardiovascular disease (CVD) risk in postmenopausal women. As a key public health strategy to reduce the incidence of CVD in the UK, dietary saturated fatty acid (SFA) intake of $\leqslant 10 \%$ of total energy is recommended ${ }^{(2)}$. The Dietary Intervention and Vascular Function-2 study aimed to investigate the effects of sequential meals of varying fat composition on postprandial vascular reactivity and associated biomarkers of endothelial function in postmenopausal women.

In an acute, double-blind, randomised, cross-over study, 32 women (mean age of $57 \pm 1 \mathrm{y}$ and BMI of $26 \pm 0.7 \mathrm{~kg} / \mathrm{m}^{2}$ ) consumed sequential mixed test meals ( 0 min, $50 \mathrm{~g}$ fat and $330 \mathrm{~min}, 30 \mathrm{~g}$ fat) rich in SFA, monounsaturated (MUFA) or $\mathrm{n}-6$ polyunsaturated (PUFA) fatty acids on 3 separate occasions, each 4-6 weeks apart. Blood samples were collected and real-time measures of vascular reactivity (flow-mediated dilatation, laser Doppler imaging, digital volume pulse) were performed before and at regular intervals after the breakfast for $480 \mathrm{~min}$.

There were no differences in the real-time measures of vascular function after the SFA, n-6 PUFA and MUFA-rich meals. A significant effect of meal fat composition was evident for the incremental area under the curve (IAUC) for the postprandial plasma nitrite (a biomarker for nitric oxide, an important vasodilator) response $(p=0.010)$, with a lower IAUC after consumption of the SFA than MUFA-rich meals $(p=0.007)$. There was also a trend for the IAUC for the nitrate and NOx (both nitrite and nitrate combined) responses $(p=0.054)$ to be influenced by the meal fatty acids, with a lower IAUC after the SFA than n-6 PUFA-rich meals $(p=0.024)$. There were significant test fat* time interactions for the time course profiles for soluble intracellular cell adhesion molecule-1 (sICAM-1) and vascular adhesion molecule-1 (sVCAM-1), with a lower sICAM-1 response after the n-6 PUFA than SFA and MUFA-rich $(p<0.001)$ meals whereas the sVCAM-1 response was different after the SFA than MUFA and PUFA-rich meals (Figure). E-selectin and P-selectin responses were not affected by meal fat composition.
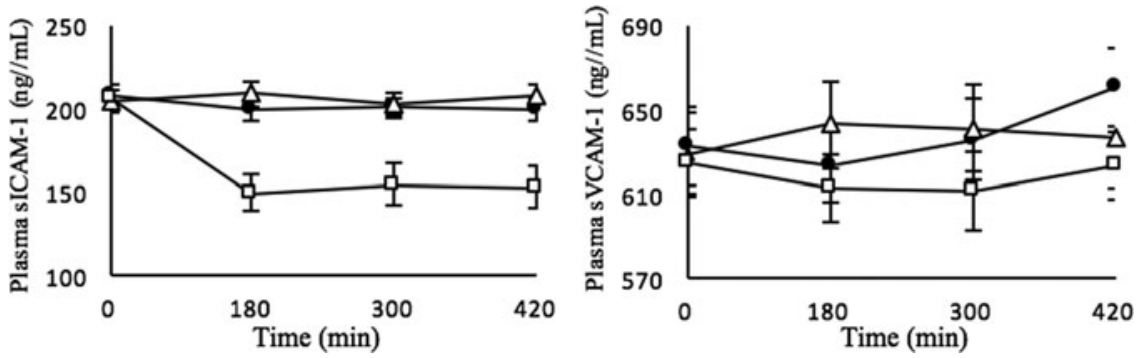

Figure. Mean \pm SEM for the sICAM-1 and sVCAM-1 responses following sequential meals $(0$ min and 330 min) enriched with SFA $(0)$, MUFA $(\triangle)$ and n-6 PUFA ( $\square$ ). Repeated measures ANOVA showed a significant test fat*time interaction $(p<0 \cdot 001)$.

In conclusion, our findings reveal a differential impact of sequential meals of varying fat composition on plasma nitrite and cell adhesion molecules (biomarkers of endothelial activation) in postmenopausal women. Further studies are necessary to examine the mechanisms underlying the effects of meal fatty acids on postprandial endothelial function.

This study was funded by UK Food Standards Agency and Department of Health Policy Research Programme (024/0036). KMR was supported by the Commonwealth Scholarship Commission, UK.

1. Davis SR, Lambrinoudaki I, Lumsden M et al. (2015) Nat Rev Dis Primers 1, 15004.

2. Do H (1991) Dietary reference values for food energy and nutrients in the United Kingdom. Report on the Panel of Dietary Reference Values of the Committee on Medical Aspects of Food Policy. London: UK. 